\title{
REFLEXIONES SOBRE LA SUPERVISIÓN CLÍNICA. UNA EXPERIENCIA DE FORMACIÓN UNIVERSITARIA
}

\section{REFLEXIONS ABOUT CLINICAL SUPERVISION. AN UNIVERSITARY LEARNING EXPERIENCE}

\author{
Daniel Fernández, Fernández. \\ Ginnette Barrantes Sáenz ${ }^{2}$
}

Fecha de recepción: 23 de agosto de 2017 - Fecha de aceptación: 15 de noviembre de 2017

\begin{abstract}
Resumen
Este artículo tiene como propósito principal presentar algunas reflexiones sobre el proceso de supervisión clínica llevado a cabo con estudiantes de psicología mediante un programa de formación clínica de la Escuela de Psicología de la Universidad de Costa Rica. El planteamiento metodológico consiste en abordar la supervisión clínica como una experiencia - acontecimiento, en donde el/la supervisor/a dirige su atención al lazo transferencial entre el estudiante y el consultante, colocándose como una tercera persona en la escena de la práctica clínica. Los resultados de esta reflexión plantean, en la misma línea que el psicoanalista Jacques Lacan, que la función secretario puede constituirse en una vía que posibilite la escucha analítica en el marco de la labor docente de supervisión clínica. A manera de conclusión, este trabajo propone que la subjetividad es una parte esencial del proceso de supervisión del programa de formación clínica descrito, en el cual el análisis de la transferencia tiene un rol preponderante.

Palabras clave: Psicoanálisis, Transferencia, Experiencia-acontecimiento, Supervisión, Función-secretario.
\end{abstract}

\begin{abstract}
The main target of this article is to present some reflections about the clinical supervision process accomplished with psychology students who participate in a professional clinical learning program of the Psychology School of the University of Costa Rica. The methodological approach entails to apprehend clinical supervision as an event-experience, where the supervisor directs the attention to the student-patient transferencial tie, placing himself/herself as a third person in the scene of the clinical practice. The results of this reflection indicate, in the same way of the psychoanalyst Jacques Lacan, that secretary function could become a way to make possible the psychoanalytical listening in the framework of the teacher work of clinical supervision. To conclude, this work propose that subjectivity is an essential part of the supervision process of the clinical leaning program described, in which the analysis of transfer has a prevailing role.
\end{abstract}

Key words: Psychoanalysis, transference, event-experience, supervision, secretary-function.

\footnotetext{
1 Escuela de Psicología, Universidad de Costa Rica, danielfernand@gmail.com

2 Escuela de Psicología, Universidad de Costa Rica, ginette.barrantes@gmail.com
} 


\section{Introducción}

Este artículo plasma algunas reflexiones en torno al proceso de supervisión clínica acaecido en el marco del dispositivo de formación universitaria "Escucharte" (2000-2013), como parte de la práctica clínica universitaria supervisada en el primer nivel de atención de los EBAIS del Área de Salud de Curridabat (Granadilla, Tirrases, Curridabat, José María Zeledón y Cipreses), a través del convenio PAIS-UCR/CCSS.

Dicha formación se inscribe en el proceso enseñanza - aprendizaje de los Módulos de Psicología de la Salud I y II ${ }^{3}$, impartidos en la Escuela de Psicología de la Universidad de Costa Rica, los cuales en ese momento forman parte del cuarto y quinto año de la carrera de psicología ${ }^{4}$ y están articulados en tres ejes fundamentales, a saber, docencia, investigación y acción social ${ }^{5}$. Durante el primer semestre se aborda la práctica clínica con niños y adolescentes y durante el segundo se recibe a los y las consultantes de la población adulta.

El Módulo, como experiencia-aprendizaje, se ubica en continuidad con los demás cursos que ofrece la carrera de Psicología en el área de la atención clínica. Su acometido fundamental es que el estudiantado pueda poner en práctica los conocimientos y habilidades profesionales recibidas hasta entonces, mediante intervenciones profesionales en las que se vincule el análisis de la realidad social y la experiencia clínica.

La inserción práctica a nivel comunitario constituye un pilar para la formación del estudiantado de Psicología, así como para el modelo de educación superior que ha caracterizado a la Universidad de Costa Rica en general y a la Escuela de Psicología en particular. Como corolario de lo anterior, la supervisión clínica representa una parte fundamental de dicho proceso, puesto que es, justamente, en esta instancia en la que convergen aspectos propios de la labor didáctica desarrollada en el salón de clase y el accionar terapéutico que se genera en el ámbito práctico - institucional ${ }^{6}$.

A continuación, se presentarán algunas reflexiones en las que se ahondará específicamente en los aspectos teórico-metodológicos que atañen a la labor de supervisión clínica del Módulo, realizada por el co-profesor y la profesora suscribientes del presente artículo, teniendo como horizonte una lectura psicoanalítica de la práctica clínica, dentro del proceso enseñanza-aprendizaje en la formación de los estudiantes de psicología de la Universidad de Costa Rica que han realizado dicho pasaje.

\section{La clínica en el tiempo de la no experiencia}

“Para qué valen los bienes de la educación si no nos une a ellos la experiencia?”. (Benjamin, 1989, p. 168)

3 En adelante se hará referencia a ambos Módulos como simplemente el Módulo, esto tanto por la facilidad a nivel referencial, como por el hecho de que a pesar de que existe una división administrativa que contempla contenidos distintos para cada uno, el abordaje teórico - metodológico que los comprende es el mismo.

$4 \quad$ En la actualidad ambos módulos se imparten en cuarto año de la carrera.

5 Estos Módulos estuvieron a cargo de la profesora Ginnette Barrantes Sáenz y de los co-profesores Daniel Fernández Fernández, Mercedes Flores González, María del Rocío Murillo Valverde, Yannina Sánchez Mora (mencionados por orden alfabético según su apellido).

6 Como requisito formal del Módulo, los y las estudiantes deben asistir a tres supervisiones semestrales con el/la supervisor/a. No obstante, el estudiantado puede solicitar las supervisiones que se requieran o bien el/la supervisor/a puede asignar más sesiones de considerarlo necesario, ya sea porque los aspectos casuísticos referidos a las personas a las se atiende lo requiriesen así, o porque la situación misma del/a estudiante/terapeuta así lo ameritase. Dichas supervisiones se solicitan por vía telefónica o por correo electrónico, tal y como se realiza en una situación profesional ordinaria de esta índole. 
Giorgio Agamben (2007), retomando un corto ensayo de Walter Benjamin en el que habla acerca de lo paupérrimo en experiencia que es la época moderna, señala que esa carencia no ha de leerse como una interpelación nostálgica a un pasado idealizado respecto a un presente cuyas experiencias serían supuestamente fútiles o insignificantes, muy por el contrario, afirma que la existencia cotidiana nunca fue más rica en acontecimientos significativos como en la actualidad. Para él, la incapacidad de traducir en experiencia esos acontecimientos, es lo que vuelve hoy, como nunca antes, insoportable la existencia cotidiana. Por experiencia entendemos no solamente un proceso de conocimiento y de adquisición de habilidades y destrezas, sino la capacidad de interrogarse sobre los aspectos inconscientes que operan como obstáculos en un lazo transferencial.

Una plétora de ejemplos cotidianos parecen dar cuenta de esta aserción benjaminiana, basta con asistir a cualquier espectáculo público para constatar con qué frecuencia los espectadores en lugar de "asistir" a la obra con su mirada, se decantan por resistir a la misma con dispositivos tecnológicos en mano; no es posible siquiera afirmar que la experiencia se volvió insustancial, pues en este caso ésta nunca existió. François Ewald y Alessandro Fontana, los editores al español de los cursos impartidos por Michel Foucault en el Collège de France, dan cuenta de una situación análoga. Refieren así que Gérard Petitjean, periodista del Nouvel Observateur, cuenta que al terminar Foucault de impartir sus lecciones en el Collège de France, "Los estudiantes se abalanzaban sobre su escritorio. No para hablarle, sino para parar los grabadores. No hay preguntas. En el tropel, Foucault está solo" (Petitjean, 1975, citado por Ewald y Fontana, 2008, p. 8) ${ }^{7}$. La experiencia requiere la inquietud de la pregunta, pero sobre todo la quietud de la respuesta.

La nulidad de experiencia no compele únicamente a los arreglos culturales contemporáneos que se expresan en lo cotidiano, sino que tiene su impronta en múltiples esferas de la sociedad, entre las cuales figura de manera privilegiada la producción y transmisión de conocimiento. Para Agamben, la expropiación de la experiencia estaba implícita en el ideario fundamental de la ciencia moderna, ya que contrario a lo que se ha repetido con frecuencia, dicha ciencia nace a partir de una desconfianza sin precedentes en relación a la experiencia tal y como era tradicionalmente entendida. Está entre saberes populares y saberes técnicos, entre saberes subjetivos y objetivos, es parte de los lenguajes que permean el dispositivo universitario de enseñanza y sobre todo al sujeto moderno confrontado con la ciencia y su subjetividad como dos modos de saber muy distintos.

La certificación científica de la experiencia que se efectúa en el experimento -que permite deducir las impresiones sensibles con la exactitud de determinaciones cuantitativas y por ende prever impresiones futuras- responde a esa pérdida de certeza que desplaza la experiencia lo más afuera posible del hombre [sic]: a los instrumentos y a los números. Pero de este modo la experiencia tradicional perdía en realidad todo valor. Porque como lo muestra la última obra de la cultura europea que todavía se funda íntegramente en la experiencia: los Ensayos de Montaigne, la experiencia es incompatible con la certeza, y una experiencia convertida en calculable y cierta, pierde inmediatamente su autoridad. (Agamben, 2007, p. 14)

Se trata pues de la experiencia como tradición perdida en donde el sujeto queda fuera de toda experiencia que no sea la medición, como lo sugiere Villacorta (2012). El punto que plantea la crítica de Agamben, es llevarnos a encontrar el fondo de la pobreza de experiencia que aleja al sujeto de la experiencia misma y lo relega a su propia desubjetivación a partir de la sobrevaloración y la verdad científica. Así, de forma opuesta a la experiencia científica que tiene un método "cierto", para Agamben la única experiencia posible del sujeto debería ser construida sobre un camino incierto o si es posible sobre una ausencia de camino.

$7 \quad$ Texto citado por Francois Ewald y Alessnadro Fontana (directores de la Edición de los cursos de Michel Foucault en el Collège de France en el Fondo de Cultura Económica), en la parte introductoria de los cursos bajo el título de "Advertencia". El artículo citado, corresponde a un artículo publicado en el Nouvel Observateur el 7 de abril de 1975, bajo el título Les Grand Prêtres de l'université française. 
Contrario a esta vía que asigna a la experiencia un carácter de movimiento sobre trayectos las más de las veces sinuosos, la psicología positivista opera bajo la máxima de un saber cuya constatación reposa en una exterioridad, es decir, mediante una instancia en la que el terapeuta participa a título de consignatario de la verdad (muy distinta de aquella posición de secretario en la escucha de un relato), misma que le es otorgada mediante la autoridad de un saber cuyo camino es siempre fijado de antemano y no admite por demás vericueto alguno. Este punto es crucial cuando él o la estudiante se enfrenta a la escucha analítica del relato y no puede (más bien no debe) escucharlo bajo la lupa de conceptos y categorías, sino que debe recibirlo mediante la atención flotante.

La supervisión desde esta perspectiva, constituye un tramo más de ese camino seguro que augura una clínica de resultados medibles y cuantificables. De esta forma, la supervisión tradicionalmente es entendida como una intervención evaluativa del desempeño del terapeuta que persigue varios propósitos: 1) un mecanismo de control de calidad de las intervenciones realizadas; 2) una vía para facilitar la eficiencia y las competencias asociadas al trabajo clínico y 3 ) un medio para brindar soporte emocional al terapeuta.

Dado que el trabajo que se lleva a cabo en los Módulos se enmarca en un dispositivo de formación universitaria, todas estas dimensiones son contempladas en los procesos de supervisión, no obstante el abordaje de las mismas apunta a derroteros distintos al control, la eficiencia, la medición y evaluación o el soporte emocional (se señala este último aspecto, pues si bien es cierto que la contención es un escenario posible dentro del proceso de supervisión, ésta última no debe pasar por un subrogado del análisis personal ${ }^{8}$ ), y por derivación adquieren un cariz diferente al propuesto por el encuadre tradicional. La supervisión universitaria no sustituye el análisis propio, ni se constituye en su paralelo, sino que procura un lugar de escucha de ese lazo como "tercera persona".

Tal y como se entiende aquí, lo que acontece en el espacio de supervisión implica un acontecimiento-experiencia cuyo principal acicate es el encuentro con lo subjetivo planteado como obstáculo, resistencia o motor en la trasferencia. En el siguiente apartado se abordará con más detalle la relación entre estos elementos.

\section{La supervisión como acontecimiento-experiencia de la transferencia}

Como en reiteradas ocasiones nos lo ha mostrado Freud, la transferencia es la materia prima del quehacer psicoanalítico y es además el punto de inflexión respecto a la tradición clínica cientificista, ya que justamente "allí está lo que separaría al analista de la posición médica o psiquiátrica: el hecho de que la "persona" de la cual él soporta los rasgos (...), será el lugar de expresión de representaciones reprimidas y, al mismo tiempo, el sesgo por el cual aparecerá lo que, en la organización del preconsciente escapa a la rememoración”. (Le Gaufey, 2006, p. 68). La supervisión concebida así, interroga al dispositivo de enseñanza pues no busca una evaluación o eficiencia del proceso de cura o de sus objetivos, sino que apunta al blanco con sus agujeros.

Bajo esta premisa, la relación transferencial deviene en un aspecto insoslayable para la labor de supervisión que se realiza en cada Módulo, verbigracia de ello, la elección del/a supervisor/a es de escogencia libre por parte de cada integrante del estudiantado. Dicho proceder resulta constitutivo del acercamiento clínico que es aquí privilegiado y marca lo que vendría a ser un primer escolio distintivo en lo que se refiere a la confluencia del quehacer psicoanalítico con el complejo ámbito de la formación

8 En relación a la labor del analista, Freud nos recuerda que "no basta que sea un individuo aproximadamente normal, debiendo más bien exigírsele que se haya sometido a una purificación psicoanalítica y haya adquirido conocimiento de aquellos complejos propios que pudieran perturbar su aprehensión del material suministrado por los analizados". (2001, p. 1657).

9 La anatomía de esta tercera persona fue descrita en un amplio estudio realizado por Guy Le Gaufey. Consultar: Le Gaufey, G. (2000). Anatomía de la tercera persona. École Lacanienne de Psychanalyse. México: Epele. 
universitaria del estudiante a nivel clínico, al cual muchas veces se homologa con el analista. Sin embargo, los tiempos del cronograma universitario están allí para recordarnos que no es posible confundir el tiempo lógico o cronológico, con el tiempo imprevisto del inconsciente.

Un segundo escolio, se relaciona con el qué del proceso de supervisión, pues a diferencia del modelo médico o de la clínica basada en la evidencia científica que conlleva inspeccionar ya sea el resultado de la anamnesis del paciente y/o auscultar el accionar del terapeuta, en el caso del Módulo, la supervisión opera en torno al lazo transferencial entre el estudiante y el consultante, como se muestra en el siguiente esquema:

Figura 1

Supervisión del lazo transferencial

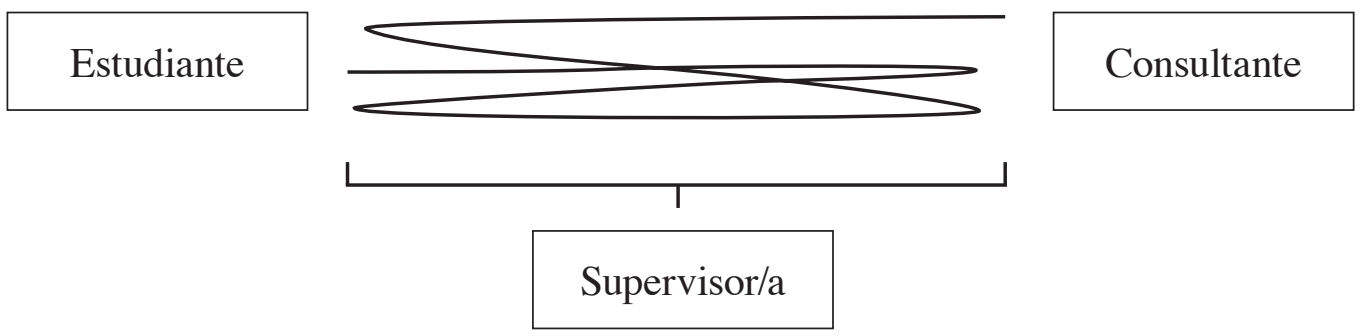

Fuente: Elaboración propia.

Conviene hacer una precisión sobre el énfasis que conlleva el abordaje aquí planteado. La constatación de cuán oportunas o inoportunas hayan podido ser las intervenciones de los y las estudiantes en las sesiones llevadas a cabo con sus respectivos consultantes, es un aspecto insoslayable por tratarse este de un proceso de formación universitaria que exige un conocimiento adecuado del marco deontológico profesional de la psicología. Sin dejar de lado esta arista, el tipo de análisis que queremos destacar en este escrito se orienta en otra dirección. La evaluación que efectúa el/la supervisor/a pasa por el hecho de asir el lazo transferencial en torno al cual son urdidas las intervenciones del estudiantado, así como por interrogar la posición de escucha como una forma de sostener una función analítica. Lo anterior implica elevar la supervisión al nivel de una experiencia en la que dicho lazo es trastocado, deslindando las singularidades de la escucha analítica.

Llevar la supervisión al plano de la experiencia, conlleva una operación compleja pero indefectible, pues como sugiere Larrosa (2002), es incapaz de experiencia aquel a quien nada le pasa, a quien nada le acontece, a quien nada le sucede, a quien nada lo toca. Examinar el lazo transferencial, supone como señala Gloria Leff (2007), advertir las marcas del tizne que deja la operación de "estar juntos en la misma chimenea”. Y 'advertir' es aquí un verbo importante, pues la supervisión no da como tarea suprimir la transferencia, sino más bien estar en la capacidad de leer sus efectos y actuar en correspondencia.

Más allá de que los y las estudiantes se apropien de técnicas y estrategias para intervenir a nivel de la consulta psicológica, se busca que puedan aprehender los corolarios que derivan de una situación terapéutica, configurando una experiencia-acontecimiento en torno a otra experiencia-acontecimiento.

La experiencia-acontecimiento es (...) lo que se opone a la repetición y al automatismo, lo que genera pensamiento y acción; a la vez, reúne y separa, enlaza y desenlaza. La experiencia transforma posiciones en el marco de una situación y en forma inmanente a ella. No hay un orden externo que se imponga y defina la situación, los lugares, las posiciones. (...) no es la recepción o la sumisión ante la imposición de forma, es la aparición de un proceso subjetivo en un determinado momento a partir de una conjunción de condiciones que lo permiten. (Greco, Pérez, y Toscano, 2008, p. 75) 
Por lo tanto, la supervisión es concebida más allá de requerimientos formales desde el punto de vista académico y curricular (aunque dichos aspectos sean efectivamente tomados en cuenta en la programática general del Módulo), procurado establecer un acercamiento clínico que brinde al estudiantado la posibilidad de (re)elaborar el decir de su propio decir.

En este punto resulta imperativo insistir en que este "efecto de decir" que se espera en dicho proceso no es elegido voluntariamente, pues el mismo toma lugar en una escena en la que el carácter de obligatoriedad no puede ser obliterado, pues se trabaja en un ámbito sujeto a un conjunto de demarcaciones propias de una matrícula universitaria. En este sentido, contrario a procurar soterrar las disparidades que derivan de esta tensión, el accionar formativo que tiene lugar en el Módulo, enfatiza precisamente el carácter ineluctable de esta relación, procurando dilucidar sus consecuencias para el quehacer clínico.

\section{La supervisión clínica y la función secretario}

"Para ser un buen secretario conviene saber muchas cosas, pero callar muchas más". (Blanc-Sánchez, 2000, p. 19)

Hasta ahora se han hecho algunas puntualizaciones sobre la forma en la que se comprende la supervisión a nivel del Módulo, precisando que la misma es entendida como una experiencia-acontecimiento que opera sobre el lazo transferencial entre el estudiante-consultante. En este apartado se brindan algunas otras presiones que atañen al rol del/de la supervisor/a dentro de este encuadre.

A pesar de los beneficios ampliamente reconocidos del sistema de salud público costarricense, en la actualidad éste afronta múltiples dificultades que afectan la prestación de servicios en general y a nivel psicológico en particular, pues como se planteó más atrás, advenimos a un contexto en el cual hay cada vez menos cabida para la experiencia. El Módulo constituye una experiencia novedosa de atención dentro de la salud tal como es entendida por la Caja Costarricense de Seguro Social (C.C.S.S), en el tanto los y las practicantes que comienzan a dar sus primeros pasos en el ámbito terapéutico, se ven confrontados a una realidad compleja que exige zanjar una serie de elementos que no pasan por el acoplamiento a un marco prescriptivo, sino más bien por la apropiación de una postura enunciativa no connivente ante la realidad que imprime la doxa científica.

Como señala Barthes:

(...) las "ciencias humanas", constituidas de manera tardía sobre el barbecho del positivismo burgués, aparecen como las coartadas técnicas que nuestra sociedad se permite a sí misma para mantener en su seno la ficción de la verdad teológica, soberbiamente -y de una manera abusiva- separada del lenguaje. (Barthes, 2009, p. 23)

Uno de los primeros obstáculos de la experiencia, surge precisamente del deseo de controlar mediante el saber académico y sobre todo ante la angustia del no saber. Cuando la técnica se convierte en un uso fetichizado en la escena clínica, el o la estudiante no tendrá otra alternativa que el recurrir a ella como tabla de salvación, ante la inercia misma que produce dicha instanciación del saber.

La asociación libre heredada por Freud, cual base fundamental del análisis, conlleva a nuestro criterio una postura enunciativa en la que la experiencia no solo resulta asequible, sino también necesaria de cara al encuentro con el otro. Freud, nos recuerda Le Gaufey, "busca con la regla fundamental confiscar la autoridad crítica, no para arrogársela, sino para disponerla de otro modo, en otra parte, en un lugar tercero donde será muy distinto tratar el asunto del análisis de la transferencia que develar su naturaleza" (2011, p. 124). De esta forma, nos vemos confrontados a un tercer escolio, que consiste en trasladar esta regla fundamental al tridente: Academia- EBAIS-Comunidad, y hacerla operar en todos sus enlaces y relaciones. 
Un aspecto esencial para hacer funcionar este engranaje, implica concebir el lugar del deseo en la escucha analítica. Lacan en su escrito La instancia de la letra en el inconsciente o la razón desde Freud (1981), destaca que el lugar de la letra en el inconsciente, es el lugar del deseo, en tanto movimiento. Es decir, el lugar del deseo que se define como deseo de otro o como deseo de deseo. La asociación libre en su radicalidad, enfrenta a quien escucha y a quien habla a esa ausencia de imagen del otro, o al llamado de un Otro como sostén de la palabra.

De esta forma, acogemos el postulado según el cual el deseo no puede ser soslayado de la escucha analítica, y es por lo tanto, conditio sine qua non del proceso de escucha en la supervisión clínica. Aquella persona sobre la que recae la labor de supervisión, debe tomar nota del deseo y de las condiciones de su efectuación, debe por lo tanto estar en la capacidad de ejercer eso que Lacan denominaba la función secretario. Una función de tercero pero cuya persona no encarna esa terceridad del lazo. Un buen secretario es, retomando un señalamiento de Jean Allouch, (2006), aquel que permite la producción de la palabra, y no sólo que permita, sino que actúa en el momento de su producción, señalando y permitiendo una decantación de significados propios al sujeto, así como un tiempo para la elaboración.

El ejercicio del secretariado en el proceso de supervisión, implica una escucha-otra, que no puede ser replegada en el orden de los parámetros apriorísticos que atañen al saber, en tanto que "el analista no lee con el saber teórico, su posición es de docta ignorancia" (Garrofe, 2000, p. 21). Este movimiento conlleva también una inflexión en lo que respecta al proceso de enseñanza-aprendizaje, pues abre una vía para asir la subjetividad en el proceso formativo, y rompe con aquella lógica que coloca al docente-clínico (una figura poco explorada en la enseñanza de la clínica) en el lugar de un preceptor que cuenta con una respuesta prefabricada para todo. Contrario a este lugar común del saber universitario, consideramos que:

\footnotetext{
El examinador siempre está sujeto a una verificación en el libro abierto, en la materialidad de cada palabra, en la curva de cada signo. (...) Esta es la razón por la que el maestro ignorante podrá, cuando tenga la ocasión, extender su competencia hasta comprobar no la ciencia del señorito instruido sino la atención que presta a lo que dice y a lo que hace. (Rancière, 2003, p. 21)
}

El maestro ignorante de Rancière, conjuga en el ámbito de la educación lo que para el psicoanálisis implica el ejercicio de la función secretario, es decir, una abstención del saber que movilice diferentes agenciamientos subjetivos y que coadyuve a la producción de sentido tanto a nivel de la escucha analítica, como del proceso de formación. Por tanto, la supervisión implicara cierto tipo de reflexividad en torno al propio decir y al propio hacer, que se inscribe concomitantemente en las coordenadas del propio deseo, lo que implica por derivación una abdicación del lado de quien supervisa, para hacer así sostenible una postura enunciativa que acoja la complejidad del sujeto y su contexto, del lado del estudiante.

\section{Reflexiones finales}

En la actualidad, el modelo de atención clínica que ofrece el sistema de salud costarricense, continúa mayoritariamente encausado a un accionar psicológico medicalizado en colusión con una medicina conductual que apunta a una clínica cada vez más ajena a la experiencia, en la cual lo subjetivo parece encontrar cada vez menos espacios de agenciamiento. La confluencia del psicoanálisis en un dispositivo de formación universitaria, es si se quiere un movimiento contra corriente, pues se insiste cada vez más en la "necesidad" de tratamientos psicológicos homogeneizados. En este sentido, rescatar un primer contacto con la experiencia de escucha analítica se convierte en una posibilidad de apertura para la elección posterior de una posición de analista.

El Módulo de Psicología de la Salud, parte de un postulado que consiste en afirmar que lo subjetivo es por definición inestandarizable, en correspondencia, el proceso formativo que se lleva a cabo 
en el Módulo apuesta por un modelo de escucha analítica que se decanta en cada una de sus facetas por este axioma programático. La supervisión clínica no es la excepción, y es por ello, que se propone abordar dicho proceso como una experiencia-acontecimiento, a partir del lazo transferencial entre el terapeuta y el consultante.

La elaboración de bitácoras y diarios de campo cumplen así una función de autobservación y escucha en la práctica que permite la interrogación de ese lazo transferencial, con lo cual se busca un efecto de reflexividad que coadyuve a aprehender los efectos significantes que suponen esos acontecimientos-experiencias. "Tomar nota", pasa de ser un ejercicio equidistante entre el cuadernillo y el estudiante, a un ejercicio en el que es perentorio "anotarse".

Como señala Pasini (2001), el acompañamiento en el proceso de atención psicológica, pone en escena distintas concepciones y modos de hacer y aprender, en la medida en que supone una oportunidad de "aprender a hacer, haciendo con". Siguiendo este planteamiento, se toma la función secretario propuesta por Lacan, como una vía para aprehender el hacer y el decir que tiene lugar en los procesos de supervisión que se desarrollan en el Módulo, dando así lugar a la producción de la palabra y a la posibilidad de elaborar los efectos del propio decir en la transferencia.

\section{Referencias}

Agamben, Giorgio. (2007). Infancia e Historia. Destrucción de la experiencia y origen de la historia. Buenos Aires: Adriana Hidalgo Editora.

Allouch, Jean. (2006). Freud y después Lacan. Ciudad de México: Epeele.

Barthes, Roland. (2009). El susurro del lenguaje. Más allá de la palabra y la escritura. Barcelona: Paidós.

Benjamin, Walter. (1989). Experiencia y Pobreza. En: Discursos interrumpidos. Filosofía del arte y de la historia. Buenos Aires: Taurus.

Blanc-Sanchez, Mireille. (2000). La palabra confiscada. En: La función secretario. Revista Litoral 25/26. École Lacanienne de Psychanalyse. México: Epeele.

Ewald, François y Fontana, Alessandro. (2008). Advertencia. En: Foucault, Michel. (Autor). Nacimiento de la biopolítica. Curso en el Collège de France (1978-1979). Buenos Aires: Fondo de Cultura Económica.

Freud, Sigmund. (2001) [1912]. Consejos al médico sobre el tratamiento psicoanalítico. Madrid: Biblioteca Nueva.

Garrofe, Pablo. (2000). Del leer en la palabra y el obstáculo musical. En: Garrofe, Pablo. (Comp.). El leer en el hablar (pp. 29-37). Buenos Aires: Grupo Editor Altamira.

Greco, María Beatriz.; Pérez, Andrea Verónica y Toscano, Ana Gracia. (2008). Crisis, sentido y experiencia: conceptos para pensar las prácticas escolares. En: Barquero, Ricardo; Pérez, Andrea Verónica y Toscano, Ana Gracia. (Comp.). Construyendo posibilidad. Apropiación y sentido de la experiencia escolar. (pp. 63-80). Rosario: Homo Sapiens Ediciones.

Lacan, Jacques, (1981). La instancia de la letra en el inconsciente o la razón desde Freud. En: Escritos I, Novena Edición. México: Siglo XXI Editores.

Larrosa, Jorge. (2002). Notas sobre a experiência e o saber de experiência. Revista Brasileira de Educação, (19).

Leff, Gloria. (2007). Juntos en la chimenea. La contratransferencia, las “mujeres analistas" y Lacan. École Lacanienne de Psychanalyse. México: Epeele.

Le Gaufey, Guy. (2000). Anatomía de la tercera persona. École lacanienne de psychanalyse. México: Epeele.

Le Gaufey, Guy. (2006). El caso inexistente. Una compilación clínica. École Lacanienne de Psychanalyse. México: Epeele.

Le Gaufey, Guy. (2011). El objeto a de Lacan. École Lacanienne de Psychanalyse. México: Epeele. 
Pasini, Vera Lucía (2001). Formação em Serviço. Ensaiando modos de ser e fazer em Saúde. En: Tomanik, Eduardo y Pires, Angela Maria. (Comp.). Psicologia Social: Desafios e Ações (pp. 42-56). Maringá: ABRAPSO.

Rancière, Jacques. (2003). El maestro ignorante. Barcelona: Editorial Laertes.

Villacorta, Claudia. (2012). Genealogía del poder y lo impolítico en Giorgio Agamben. (Tesis de Maestría). Universidad Centroamericana José Simeón Cañas, Antiguo Cuscatlán, El Salvador. 
